\title{
REDES SOCIALES Y APLICACIONES DE MÓVIL: USO, ABUSO Y ADICCIÓN
}

\author{
Ana Isabel Isidro de Pedro \\ Profesora Titular de E.U. \\ Departamento de Psicología Social y Antropología. \\ Universidad de Salamanca \\ anyis@usal.es \\ Tamara Moreno Martín \\ Educadora Social \\ tamaraedsocial@gmail.com
}

Fecha de Recepción: 17 Febrero 2018

Fecha de Admisión: 10 Abril 2018

\section{RESUMEN}

La abrumadora popularidad de las Redes Sociales y las aplicaciones del móvil es un fenómeno ampliamente generalizado que tiene innegables implicaciones educativas. De hecho, se han convertido en herramientas casi imprescindibles en la vida de las personas. Y es, precisamente, en esta incuestionable e inquietante realidad donde hemos hallado la justificación para llevar a cabo el presente trabajo, cuyo objetivo es conocer la relación que las personas tienen con las Redes Sociales y aplicaciones móviles -destacando el WhatsApp-pudiendo pasar ésta de un simple uso a un manifiesto abuso e, incluso, adicción a las mismas. Se pretende inquirir el uso que se hace de dichas herramientas y las consecuencias negativas que pueden acarrear cuando aparece un uso abusivo. Así, además del potencial deterioro de las relaciones interpersonales, las habilidades sociales y la capacidad de comunicación, hemos investigado posibles problemas de control y pérdida de intimidad que pueden derivarse, especialmente en el ámbito de la pareja, pudiendo pergeñar los primeros pasos en la violencia de género. Para ello, hemos utilizado una metodología cuantitativa, empíricoanalítica. El instrumento de medida utilizado es un cuestionario ad hoc, de elaboración propia, constituido por 56 ítems, que fue aplicado a una muestra final de $n=226$ sujetos. Los resultados permiten concluir que el uso de estas herramientas es masivo, así como la frecuencia general con la que se utilizan, lo que supone una cierta limitación en la vida de las personas, dificultando específicamente aspectos de su vida social, pudiendo conllevar, efectivamente, problemas añadidos, como el uso arriesgado de las posibilidades de control que presentan tales herramientas.

Palabras clave: redes sociales; WhatsApp; móvil; relaciones sociales; relaciones interpersonales; control; comunicación 


\title{
REDES SOCIALES Y APLICACIONES DE MÓVIL: USO, ABUSO Y ADICCIÓN
}

\begin{abstract}
Social networks and mobile applications: use, abuse and addiction.

The overwhelming popularity of Social Networks and mobile applications is a widespread phenomenon that has undeniable educational implications. In fact, they have become almost indispensable tools in the lives of people. And it is precisely in this unquestionable and disturbing reality where we have found the justification to perform this work, whose objective is to know the relationship that people have with Social Networks and mobile applications -emphasizing WhatsAppwhich could pass from a simple use to a manifest abuse and, even, addiction to them. It is intended to inquire the use made of these tools and the negative consequences that can result when an abusive use appears. Thus, in addition to the potential deterioration of the interpersonal relationships, the social skills and the communication capacity, we have investigated possible problems of control and loss of intimacy that may arise, especially around the couple, and can be the first steps in gender violence. For that, we have used a quantitative empirical-analytical methodology. The measurement instrument used is an ad hoc questionnaire, prepared by the authors, consisting of 56 items, which was applied to a sample of $n=226$ individuals. The obtained results allow us to conclude that the use of these tools is massive, as well as the general frequency with which they are used, what supposes a certain limitation in the life of the people, specifically complicating aspects of their social life, being able to effectively involve added problems, such as the risky use of the control possibilities presented by such tools.
\end{abstract}

Keywords: social networks; WhatsApp; mobile; social relationships; interpersonal relationships; control; communication

\section{ANTECEDENTES}

El presente trabajo se ha llevado a efecto para intentar conocer, con un cierto grado de rigor y precisión, la relación que las personas tienen en la actualidad con las Redes Sociales y aplicaciones que sus teléfonos móviles ofrecen, pudiendo pasar ésta del simple uso a un manifiesto abuso e, incluso, adicción a las mismas. No obstante, lo que realmente nos interesa no es determinar o 'diagnosticar' si existe un abuso o una adicción, sino conocer el uso que se da a estas herramientas y las consecuencias negativas que pueden acarrear en la vida de las personas cuando aparece una dependencia, especialmente en lo que a la relación con los demás se refiere o cuando se realiza un mal uso de ellas, incluyendo problemas de control y de pérdida de intimidad. Por último, trataremos el potencial deterioro de relaciones, habilidades sociales y capacidad de comunicación producido como consecuencia de relaciones virtuales, a través de una pantalla, cada vez más frecuentes, que van en detrimento de las relaciones presenciales.

Así, con la aparición de los Smartphone o teléfonos inteligentes, los móviles se han convertido en una parte fundamental de nuestras vidas, a partir de los cuales no solo nos podemos comunicar telefónicamente a la manera tradicional, sino también a través de nuevas aplicaciones, cómo WhatsApp, o de las propias Redes Sociales a las que podemos no solo conectarnos desde el propio teléfono, sino instalarlas y recibir las notificaciones y avisos de novedades al momento, como si de una llamada o mensaje instantáneo se tratase. Es decir, el móvil se ha convertido en un importantísimo elemento de socialización que nos ofrece un abanico casi infinito de posibilidades, mediante el cual tenemos a nuestro alcance alternativas de ocio, comunicación y ocio prácticamente ilimitadas. Esta fascinante realidad puede Ilegar a suponer un gran problema, pudiendo llegar a convertirse en una adicción 0 , al menos, en un uso abusivo cuando, de alguna manera, comienza a limitar y condicionar nuestras vidas por ser utilizado en exceso o por interferir y condicionar actividades cotidianas, laborales, sociales, familiares 0 , incluso, por perjudicar la salud física del individuo sin capacidad de control sobre la situación. 
Centrándonos concretamente en WhatsApp, se trata de una aplicación gratuita de mensajería instantánea que se puede instalar en el smartphone y que, actualmente, su popularidad es tal que se ha convertido en una herramienta obligatoria en cualquier teléfono.

Avanzando un paso más, es importante incluir el concepto de 'nomofobia', término derivado de no mobile phone phobia. Este concepto, según publica Martínez (s.f.), supone un trastorno que sufre, sin saberlo, la mitad de la población, caracterizado por un fuerte estado de ansiedad o miedo irracional a estar sin el móvil.

En este sentido, es importante indicar qué se entiende por adicciones comportamentales. El Centro de Investigaciones Médicas en Ansiedad (s.f.) las define de la siguiente forma: "se llama así a una serie de trastornos que se ubican entre las adicciones y el trastorno obsesivo compulsivo, vinculados a actividades humanas diversas, a menudo placenteras, no relacionadas con la ingestión de sustancias químicas" (s. p.).

Según indica Echeburúa (2012a), las adicciones no-químicas suponen la realización de conductas repetitivas, con el objetivo de aliviar tensiones a través de comportamientos que pueden resultar contraproducentes para el sujeto. La urgencia por realizar y completar dichas conductas y su malestar si no se pueden llevar a cabo se parecen al síndrome de abstinencia sufrido por los drogodependientes. "Las personas pueden hacerse adictas a cualquier conducta placentera, pero hay ciertas características en el uso de Internet que lo hacen particularmente adictivo" (p. 435). En tal sentido, Echeburúa (2012b) sostiene que la adicción a las Redes Sociales supone la búsqueda, a través de ellas, del alivio de un malestar emocional, intentando ahuyentar el aburrimiento, la soledad, la ira 0 el nerviosismo.

Sin embargo, "se observa una indefinición conceptual sobre los conceptos de abuso y adicción al móvil, gran disparidad en la adopción de criterios diagnósticos y multiplicidad de instrumentos para su estimación" (Pedrero, Rodríguez y Ruíz, 2012, p. 139). Por lo que no existe una definición ni diferenciación clara que marque cuándo se trata de un mero uso, entrando dentro de lo "normal" o cuándo, por el contrario, puede pasar a abuso 0 adicción, ya sea respecto al móvil 0 a internet en general.

Así las cosas, diferentes autores etiquetan de manera distinta ciertas situaciones, pero la literatura está prácticamente de acuerdo en que el abuso y la adicción implican no tanto el tiempo dedicado, sino el grado en el que se dejan de lado otras tareas cotidianas.

Existe constancia de que el uso inadecuado y sin control de la telefonía móvil puede causar problemas comportamentales, afectivos y sociales y se caracteriza por los mismos elementos que el Diagnostic and Statistical Manual of Mental Disorders, DSM-IV-TR enumera para la consideración de una conducta como trastorno adictivo: síntomas de abstinencia, falta de control, problemas derivados del uso y la tolerancia e interferencia con otras actividades (Chóliz, 2010).

Y, por su parte, para Echeburúa y Corral (2010) "el uso y abuso de internet están relacionados con variables psicosociales, tales como la vulnerabilidad psicológica, los factores estresantes y el apoyo familiar y social" (p. 91).

En otro orden de cosas, las Redes Sociales presentan también otros riesgos, entre los que Echeburúa (2012b) incluye el acoso, la pérdida de intimidad y el acceso a contenidos inapropiados. Centrándonos concretamente en el problema de la pérdida de intimidad, explica cómo la estructura de las Redes Sociales crea la confusión entre lo íntimo, lo privado y lo público.

En este sentido, Sarrió y Soler (2015) apuntan a la posibilidad de perder la privacidad al manejar de manera poco adecuada la información que se muestra y exhibe en las redes, teniendo en cuenta que una vez que se "cuelga" se pierde el control y prácticamente cualquier persona puede acceder a ella. 


\section{REDES SOCIALES Y APLICACIONES DE MÓVIL: USO, ABUSO Y ADICCIÓN}

Por su parte, las posibilidades de control que se derivan concretamente del uso de WhatsApp, son posibles, cómo indica Ricou (2012), mediante la activación de herramientas como la última conexión y Tic azul para saber cuándo se han leído los mensajes enviados. Existe la posibilidad de no activar ninguna de estas opciones, de forma que el resto no pueden controlarte de esta forma; sin embargo, como señala el mismo autor, la mayoría activa estas opciones, pues pesa más la curiosidad por saber qué hacen nuestros contactos, que cuidar el hecho de que sepan de nosotros. Asegura, además, que algunos psicólogos empiezan a detectar rupturas en las parejas por motivos como conexiones a deshoras que no se pueden explicar y conductas obsesivas al utilizar WhatsApp.

Como es común en situaciones de control hacia el otro, se genera desconfianza y se sistematizan este tipo de conductas obsesivas muy peligrosas en cualquier relación, incluso cuando ésta ya ha terminado.

Por su parte, Herrera-Gayosso (2016) presenta la evolución de la comunicación de las parejas desde aquella época, aún cercana, en que la única manera que había era llamar por teléfono a casa $y$, en el caso de no encontrar a la persona, volver a intentarlo en otro momento hasta ahora, en que, con la generalización de las nuevas tecnologías, se puede estar constantemente en contacto, eliminando prácticamente la frontera entre la relación de pareja y el derecho a la privacidad personal.

No obstante, el problema no está en las posibilidades que otorgan los avances tecnológicos, sino en la mala interpretación y el uso disfuncional de los mismos. Cuando una persona utiliza esta herramienta con un objetivo primordial de control -en este caso dirigido a la pareja- se disparará la ansiedad de este individuo. Además, bajo estas motivaciones inadecuadas, generadas y generadoras de celos irracionales, subyace una gran inseguridad personal.

En este sentido, existen dos cuestiones que influyen en el uso del WhatsApp como medio de control, a saber: la percepción errónea (que permite tener un primer conocimiento basado en impresiones pero que, unido a la falta de información real, puede llevar con facilidad a una equivocación) y la asunción (debida a la inseguridad y desconfianza, por la cual se terminan tejiendo una serie de ideas poco racionales, asumiendo que los peores temores -cómo el engaño- son ciertos) (HerreraGayosso, 2016).

Finalmente, y por lo que se refiere a las relaciones interpersonales y la capacidad de comunicación, Cornejo y Tapia (2012) señalan que, en la vida cotidiana, están en constante transformación, lo que ha sido potenciado exponencialmente por las nuevas aplicaciones tecnológicas, generando profundos cambios en la comunicación interpersonal. "El hecho de que las interacciones digitales entre las personas se incrementen tanto, está creando una percepción distinta del espacio y el tiempo, una sensación de inmediatez de los acontecimientos y una aceleración en los procesos” (p. 225).

De este modo, es posible informar a tiempo real de cualquier cosa. Ya apenas se espera para quedar con alguien y hablar, sino que se vehicula todo a través de una pantalla, pudiendo hacerlo en cualquier momento, como sustituto de la comunicación interpersonal real, lo que genera a la vez un deterioro de las habilidades sociales y de la capacidad de comunicación.

\section{OBJETIVOS}

Objetivos generales:

Comprobar el grado de uso y la relación que las personas tienen en la actualidad con las Redes Sociales y aplicaciones móviles, destacando el WhatsApp.

Detectar posibles problemas y consecuencias negativas en la vida de los consumidores a causa del uso abusivo.

Objetivos específicos:

Averiguar la posible dependencia que las personas tienen de las Redes Sociales y el móvil.

Conocer la frecuencia con la que se utilizan el móvil y las Redes Sociales. 
Detectar potenciales problemas de control sobre los demás al utilizar de manera torticera las posibilidades que presentan las Redes Sociales y, en especial, el WhatsApp.

Relacionar el posible uso de las Redes Sociales y el WhatsApp con un deterioro de la comunicación interpersonal.

\section{METODOLOGíA}

La presente investigación se llevó a efecto a través de un cuestionario ad hoc, de elaboración propia, constituido por 55 ítems que fue aplicado a una muestra final integrada por 226 sujetos -todos ellos mayores de 18 años- que completaron el cuestionario de manera on line.

Una vez rellenados todos los formularios, incluimos los datos en el paquete de análisis estadístico SPSS, mediante el cual se trataron y analizaron.

\section{RESULTADOS Y DISCUSIÓN}

En primer lugar, las Figuras 1a y 1b, representan la distribución de la muestra por sexo y edad respectivamente, de donde podemos apreciar que la proporción de mujeres es notablemente superior, suponiendo un $75,7 \%$ de la muestra, así como la proporción de encuestados que se encuentran en la franja de edad de entre 18 a 25 años, con un 68,3\%, de modo que nuestra investigación se basa en datos especialmente centrados en una población adulta joven, aunque también teniendo en cuenta otros sectores de la sociedad de mayor edad que también utilizan asiduamente estas nuevas formas de comunicación.

Figura 1a. Distribución de la muestra por sexo

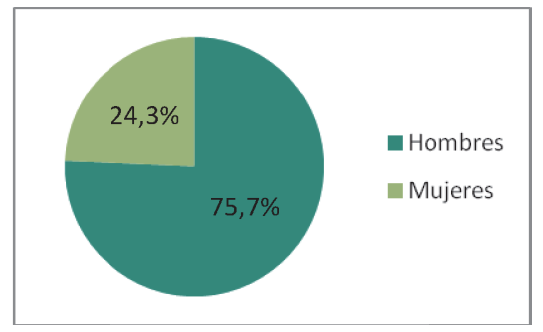

Figura 1b. Distribución de la muestra por edad

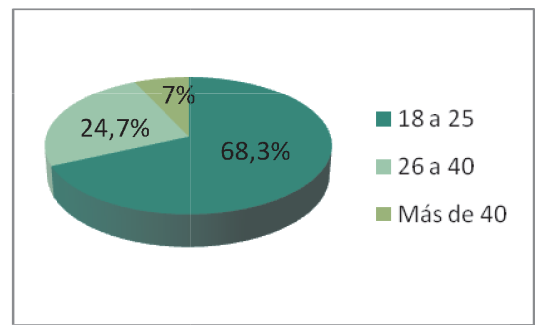

A modo general, señalar que el $100 \%$ de la muestra utiliza el WhatsApp, afirmando el $97,8 \%$ que tienen activados los datos móviles y están siempre conectados. Además, el 97,4\%, admite tener uno 0 varios perfiles en Redes Sociales, de los cuales el 76,47\%, dice tenerlas instaladas en sus móviles.

Como ya hemos señalado, en la actualidad el móvil parece una extensión de nosotros mismos y es frecuente ver a la gente interactuando con su teléfono móvil. De hecho, como podemos ver en las Figuras 2a y 2b, los sujetos de la muestra (90\%) siempre llevan encima el móvil cuando salen de casa. Además, se preguntó si volverían a casa a por el móvil en caso de olvidarlo, a lo que el $30,9 \%$ respondió que sí, lo que justifican con una posible necesidad de llamar a alguien o por entretenimiento, dadas sus múltiples posibilidades. Tan solo el 18,7\% afirmaron que no volverían a por él. Por su parte, las personas restantes respondieron que tal vez, constituyendo éstas una mayoría, añadiendo que depende de la situación o del tiempo que vayan a pasar fuera de casa. 
Figura 2a. Porcentaje que siempre lleva el móvil

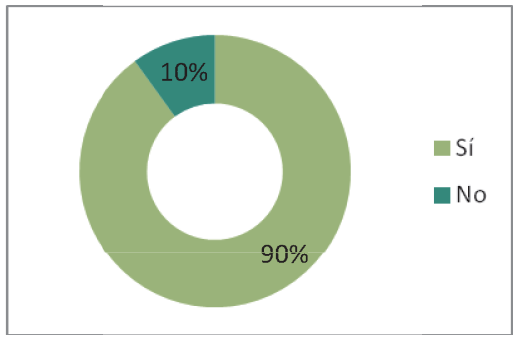

Figura 2b. Porcentaje que volvería a por el móvil

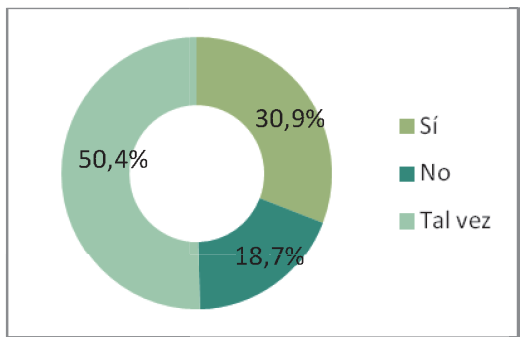

Pero lo que más llama la atención de estos datos es cómo ante la cuestión de si siempre llevan el móvil encima, un importante $90 \%$ de las personas reconoce que sí (al fin y al cabo es un hecho objetivo: o lo llevas o no) y, sin embargo, en caso de olvidarlo solo un 30,9\% reconoce firmemente que volvería a por él, mientras el resto dice no necesitarlo o solo si es una situación de urgencia 0 necesidad por estar lejos de casa. Podemos preguntarnos entonces ¿por qué razón, conscientemente, siempre lo llevan consigo? Conjeturamos que la respuesta a esta posible incongruencia quizá radique en el factor de deseabilidad social, ya que llevar el móvil contantemente es algo socialmente aceptado y, de facto, una acción común y, sin embargo, reconocer volver a casa a por el móvil porque no puedes estar sin él implica reconocer una cierta dependencia, por lo que muchos pueden esconderse detrás del "tal vez".

En la misma línea, encontramos incongruencias en las Figuras $3 a$ y 3b, donde el 86,8 \% de la muestra asegura ser capaz de estar un día entero sin su teléfono móvil y además indican en su mayoría (30,4\%) que no les afectaría y que se sentirían indiferentes, frente al $28,6 \%$, el $15,4 \%$ y el $11 \%$ que reconocen que se sentirían desubicados, incómodos y ansiosos respectivamente. Por su parte, el 68,7\% piensa que podría estar una semana sin WhatsApp. Sin embargo, aunque la mayoría dice no importarle, acabamos de ver cómo un elevado porcentaje lo tiene siempre encima y tan solo una minoría aseguraba no volver a por el móvil en caso de olvidarlo.

Figura 3a.

Porcentaje capaz de pasar un día sin móvil

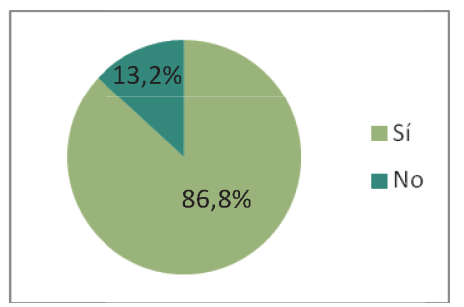

Figura $3 b$.

Porcentaje capaz de pasar una semana sin WhatsApp

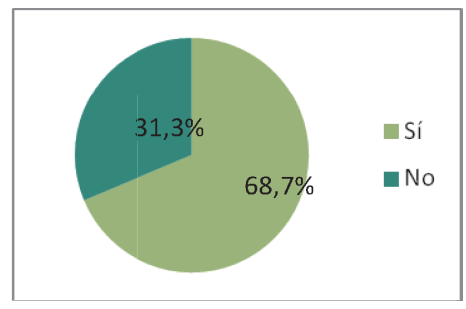


Figura 4. Porcentaje que tiene activada la última conexión y Tic azul en WhatsApp

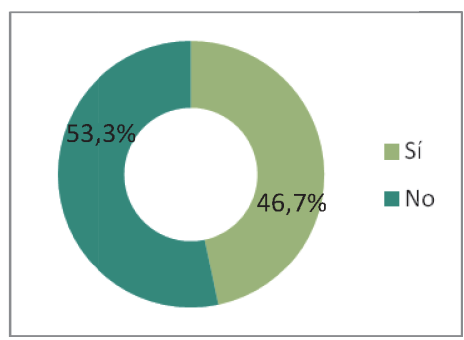

Pasando a las posibilidades de control y en relación a la opción disponible en la aplicación de WhatsApp de activar o no tu última conexión y tic azul, podemos observar en la Figura 4, cómo casi la mitad de los encuestados afirman tener activadas ambas opciones (46,7\%).

En la práctica esto supone una pérdida de intimidad, al conocer los demás cuándo ha sido la última vez que has entrado a ver tus mensajes o en qué momento has leído los que te han enviado.

Obviamente, el problema en sí no está en las posibilidades que ofrecen estas opciones, sino en la forma de utilizarlas. Con frecuencia son usadas como argumento para exigir una respuesta inmediata, generando disputas en las relaciones debidas a celos derivados de ello y "obligando" a la otra persona a hablar en un momento que quizá no pueda 0 , simplemente, no quiera hacerlo.

En relación a esto, podemos ver en la Figura 5a, cómo aproximadamente el 19,2\% de los sujetos reconocen tener habitualmente problemas con sus amigos o su pareja por no contestar de manera inmediata a sus mensajes, mientras que entre el $11,6 \%$ acepta haber tenido alguna vez estos problemas. Lo que quiere decir que casi un tercio de la muestra ha sufrido, al menos ocasionalmente, las consecuencias negativas de esta posibilidad de control del otro que proporciona el WhatsApp.

Por su parte, comparando estos resultados con la Figura 5b, llama la atención cómo el 28\% afirma preocuparse 0 enfadarse con frecuencia si es a él a quien los demás no le contestan y el $33,8 \%$ reconoce haberlo hecho alguna vez. Lo cual supone un porcentaje significativamente mayor de personas que reconocen haberse molestado si alguien no le contesta que el porcentaje de aquéllos que reconocen haber llegado a tener problemas con otros por este motivo. De ello, se podría deducir que, en muchos casos, se asume como algo normal, sin llegar a considerarlo un inconveniente para las relaciones, pudiendo ocupar el control y el recelo una posición perfectamente asumida, integrada y normalizada en las conversaciones y las relaciones.

Figura 5a. Porcentaje que ha tenido alguna vez problemas con su pareja o amigos por no contestar inmediatamente a sus WhatsApp

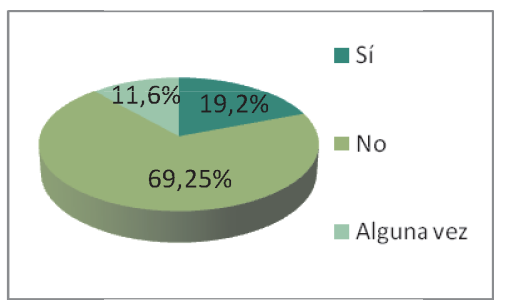


Figura 5b. Porcentaje que se ha enfadado o preocupado si otros no contestan a sus mensajes estando "en línea"

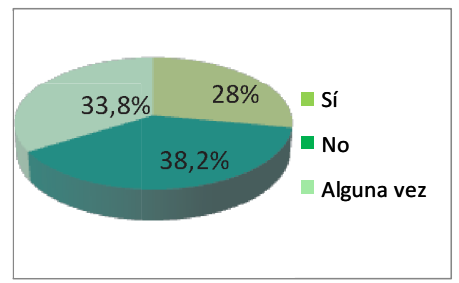

Por su parte, en la Figura 6 observamos el uso que se da al WhatsApp en relación al tema del control, pudiendo eliminarse la barrera entre la relación en pareja y la propia intimidad, sin ser necesarias las herramientas anteriormente nombradas (aunque de esa forma se acucie el problema).

Figura 6. Porcentajes referidos a posibles situaciones de control con la pareja

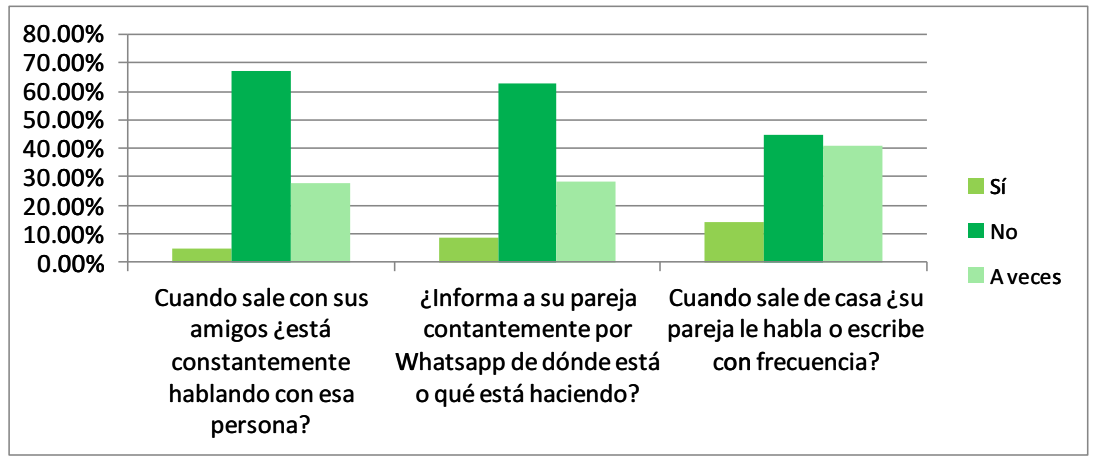

Aquí, podemos ver cómo un $5 \%$ admite estar hablando siempre con su pareja mientras se encuentra con sus amigos, seguido de un importante $27,6 \%$ que admite haber realizado a veces esta acción. Son cifras realmente preocupantes, teniendo en cuenta que un tercio de la muestra confirma esta acción, habiendo dejado de lado la comunicación interpersonal directa con otras personas.

En la cuestión de si informan constantemente a su pareja de dónde están o qué están haciendo, la mayoría $(62,6 \%$ ) lo niega, aunque el 8,8\% reconoce hacerlo siempre y el $28,6 \%$ hacerlo a veces.

Por último, en la tercera pregunta referida a si su pareja le habla o escribe con frecuencia mientras está fuera de casa, la respuesta del $44,7 \%$ de los integrantes de la muestra es negativa. No obstante, los porcentajes de las otras dos respuestas afirmativas suman mayor puntuación, constituyendo el 'Sí' un $14,3 \%$ y el 'A veces' un $41 \%$.

$Y$ dado que en las preguntas anteriores destaca la negación, es curioso apreciar cómo parece tenderse a ser más sincero cuando la pregunta no incluye directamente como "ejecutor" de la acción a la persona que responde. En este caso, resulta más fácil reconocer que es el otro miembro de la pareja quien interacciona constantemente, ya que es una forma de eludir responsabilidad, pero no tanto asumir que uno mismo cede de alguna manera y habla con el otro constantemente o le informa de lo que hace ya que, en este caso, el problema puede ser también propio. 
Por último, en la Figura 7, podemos atisbar un posible deterioro de la comunicación interpersonal como consecuencia del uso del móvil.

Figura 7. Grado de interacción con el móvil mientras se está con otras personas

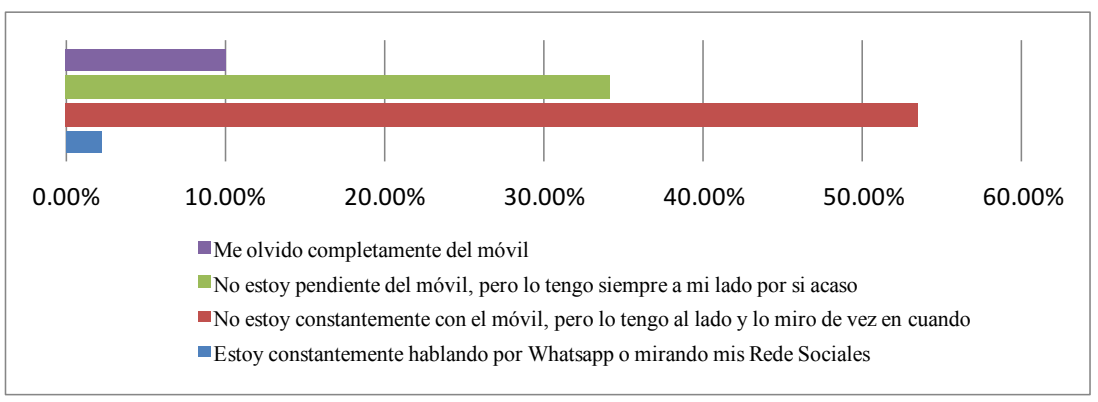

Vemos cómo solo un pequeño porcentaje de sujetos $(2,2 \%)$ admite estar constantemente mirando sus Redes Sociales o hablando por WhatsApp mientras están con otras personas. Mientras que la gran mayoría (53,5\%) afirma no estar constantemente con el móvil, pero sí tenerlo siempre cerca, mirándolo de vez en cuando; seguido de un $34,2 \%$ que asegura no estar pendiente de él, pero sí tenerlo siempre cerca por si acaso, lo que, de alguna manera, puede considerarse una necesidad. Asimismo, solo un escueto $10,1 \%$ del total, dice ser capaz de olvidarse completamente del móvil cuando está relacionándose con otras personas. De ello podemos deducir que alrededor del $90 \%$ de los encuestados están, de alguna manera, deteriorando su comunicación directa con el otro teniendo, en mayor o menor medida, una dependencia respecto al móvil y no siendo capaces de centrarse únicamente en la comunicación personal cara a cara que están teniendo con otra u otras personas de forma presencial.

Finalmente y en la misma línea, ahora no desde una perspectiva meramente personal sino colectiva, una amplísima mayoría confiesa que cuando queda con sus amigos, cada cual está pendiente de su móvil y hay ratos que ni siquiera nadie habla entre sí, reconociendo que esto pasa frecuentemente $(21,4 \%)$ o a veces $(63,3 \%)$. Tan solo el $14,8 \%$ de los sujetos asegura que esa situación nunca se produce en su grupo de amigos.

\section{CONCLUSIONES}

No podemos, ni pretendemos, etiquetar de abuso o adicción los comportamientos de los sujetos de la muestra en cuanto al uso que hacen de las Redes Sociales y el WhatsApp. Sin embargo, de acuerdo con los resultados obtenidos, sí podemos afirmar que su uso es bastante elevado, así como también es alta la frecuencia general con la que se utilizan. De este modo, estas herramientas se han convertido en una parte importante de la vida cotidiana de las personas teniendo, casi constantemente, el móvil encima y estando, en muchos casos, conectados siempre a las redes. Y, por ende, esto puede suponer una limitación importante en sus vidas, generando dependencia y obstaculizando, perjudicando o dificultando otros aspectos de su vida social.

Además de la adicción que puede generar -lo que acarrea consecuencias semejantes a las producidas por la adicción a sustancias-, un uso poco responsable de estas aplicaciones puede conllevar otros riesgos añadidos, como el control del otro y hacia el otro. Así, el control hacia otras personas implica un riesgo importante en la relación y más específicamente para la persona que lo sufre -como sucede en los casos de violencia de género- y maximiza este peligro el hecho de que 


\section{REDES SOCIALES Y APLICACIONES DE MÓVIL: USO, ABUSO Y ADICCIÓN}

las Redes Sociales (y específicamente WhatsApp) ofrezcan opciones añadidas que permiten la posibilidad de estar en contacto continuo e incluso conocer dónde y cuándo la otra persona se ha conectado. De este modo, muchas parejas están casi en constante contacto, incluso cuándo alguno de los miembros se encuentra con sus amigos o familia, elevando las posibilidades de generarse dudas, celos y discusiones gratuitas en el caso de no contestar con inmediatez a los mensajes. Motivo por el cual también se generan malestares y enfados entre amigos y conocidos. La cuestión es que tales opciones se han convertido en herramientas tan cotidianas en la vida de las personas que este tipo de conductas están asumidas y normalizadas y, en muchos casos, no son reconocidas como un problema.

\section{REFERENCIAS BIBLIOGRÁFICAS}

Barrios, H. (2016, enero 22). Selfies, narcisismo y nomofobia. El nuevo diario. Recuperado 14 de marzo de 2017, de http://www.elnuevodiario.com.ni/opinion/382910-selfies-narcisismo-nomofobia/

Centro de Investigaciones Médicas en Ansiedad (s.f.). Trastornos de ansiedad. Recuperado 5/4/2017, de: http://www.centroima.com.ar/trastornos_de_ansiedad_adicciones_comportamentales.php

Chóliz, M. (2010). Mobile phone addiccion: A point of issue. Addiction, 105, 373-374. doi: 10.1111/j.1360-0443.2009.02854.x.

Cornejo, M. y Tapia, L. M. (2012). Redes Sociales y relaciones interpersonales en Internet. Fundamentos en Humanidades, 2 (24), 219-229.

Echeburúa, E. (2010). Adicción a las nuevas tecnologías y a las Redes Sociales en jóvenes: Un nuevo reto. Revista Adicciones, 22(2), 91-96.

Echeburúa, E. (2012a). Factores de riesgo y factores de protección en la adicción a las nuevas tecnologías y redes sociales en jóvenes y adolescentes. Revista Española de Drogodependencias, 37(4), 435-447.

Echeburúa, E. (2012b). Adicción a las redes sociales y nuevas tecnologías en niños y adolescentes (1ํe ed.). Madrid: Ediciones Pirámide.

Echeburúa, E. y de Corral, P. (2010). Adicción a las Nuevas Tecnologías y a las Redes Sociales en jóvenes: Un nuevo reto. Revista Adicciones, 22(2), 91-96.

Herrera-Gayosso, V. (2016). Whatsapp y parejas: relaciones de amor basadas en la ansiedad. Recuperado de http://psicocode.com/psicologia/whatsapp-y-pareja-relaciones-de-amor-basadas-en-la-ansiedad/

Martínez, L. (s.f). ¿Qué es la nomofobia?. Revista Muy Interesante. Recuperado de https://www.muyinteresante.es/curiosidades/preguntas-respuestas/que-es-la-nomofobia151392813381

Pedrero, E. J., Rodríguez, M. T. y Ruíz, J. M. (2012). Adicción o abuso del teléfono móvil. Revisión de la literatura. Revista Adicciones,24(2), 139-152.

Ricou, J. (2012, noviembre 4). Cómo afecta el Whatsapp a las relaciones de pareja. La Vanguardia. Recuperado 13 de abril 2017, de: http://www.lavanguardia.com/vida/20121104/54354818574/whatsapp-relaciones-pareja.html

Sarrió, C. y Soler, A. (2015). ¿Sustituyen las Redes Sociales a las relaciones interpersonales? Recuperado de http://www.gestalt-terapia.es/sustituyen-las-redes-sociales-las-relaciones-interpersonales/ 